\title{
Praxisorientierte Psychotherapieforschung - Leitfaden zur Förderung von Wissenschaft und Forschung in der psychotherapeutischen Ausbildung
}

\author{
Gabriele Riess
}

Angenommen: 5. Mai 2021 / Online publiziert: 11. Juni 2021

(c) Springer-Verlag GmbH Austria, part of Springer Nature 2021

\section{Psychotherapie leistet Wichtiges und ist dennoch keine selbstverständliche Versorgungsleistung im Gesundheitssystem}

Psychotherapie ist eine zentrale Versorgungsleistung im Gesundheitssystem, sie fördert die selbstbestimmte Entwicklung von Subjekten im Sinne ihrer psychischen Gesundheit. Dass letztere einen entscheidenden und nicht zu vernachlässigenden Faktor einer gesunden Bevölkerung darstellt, zeigen auch die Folgen der psychischen Belastungen durch die CoronaPandemie bzw. deren Bekämpfung. Gleichzeitig ist das gesellschaftliche Bewusstsein für die Bedeutung von Psychotherapie erstarkt. Dieser Umstand spiegelt sich aber nicht unbedingt wider in der Position, die der Psychotherapie im Gesundheitssystem eingeräumt wird. Langfristig muss Psychotherapie - parallel zur somatisch-medizinischen Versorgung - jenen Stellenwert erhalten, der ihrem Nutzen entspricht. Dabei kann Psychotherapieforschung helfen: Sie zeigt den Bedarf an dieser zentralen Versorgungsleistung auf, belegt und verbessert deren Qualität, Effektivität und Effizienz und sichert so den Wert, den diese für Patientinnen und Patienten hat. Damit rückt Psychotherapie stärker in den Fokus des Versorgungsinteresses, was wiederum die Position der Berufsgruppe stärkt. Das Bundesministerium für Soziales, Gesundheit, Pflege und Konsumentenschutz (BMSGPK) setzt daher eine entsprechende Initiative zur Förderung von Wissenschaft und Forschung in der Psychotherapie.

\section{G. Riess $(\bowtie)$}

Koordinationsstelle Psychotherapieforschung an der Gesundheit Österreich GmbH, 1010 Wien, Österreich gabriele.riess@goeg.at

\section{Eine Koordinationsstelle für Psychotherapie- forschung als Brückenfunktion zwischen Ausbildung, Forschung und Praxis}

Das BMSGPK möchte Wissenschaft und Forschung, wie sie in den Nachbardisziplinen bereits lange Tradition haben, auch in der Psychotherapie ${ }^{1}$ stärken und plant, die Aktivitäten zu Wissenschaft und Forschung der psychotherapeutischen Ausbildungsinstitutionen (im Verfahrenscluster ${ }^{2}$ ) in Zukunft regelmäßig zu erfassen. Es versteht seine Initiative vor dem Hintergrund der Qualitätssicherungsziele des Psychotherapiebeirats $^{3}$. Das Bundesministerium beauftragte daher die Koordinationsstelle Psychotherapieforschung an der Gesundheit Österreich $\mathrm{GmbH}$ (eingerichtet im Jahr 2012) mit der Entwicklung von Empfehlungen zur Förderung von Wissenschaft und Forschung in der psychotherapeutischen Ausbildung, die auf einem spezifischen Stufenplan zur Förderung von Wissenschaft und Forschung basieren.

Die Koordinationsstelle Psychotherapieforschung hat die Aufgabe, die nationale Psychotherapieforschung bzw. eine forschungsfreundliche Kultur insbesondere in den psychotherapeutischen Ausbildungsvereinen $-\mathrm{zu}$ fördern sowie eine Brücke zu schlagen zwischen klinisch-praktischer Erfahrung und wissenschaftlich-akademischer Forschungskompetenz. Sie wird begleitet und beraten von einem

\footnotetext{
${ }^{1}$ Die Berufsumschreibung in $\S 1$ Psychotherapiegesetz hält fest, dass die psychotherapeutische Behandlung mit wissenschaftlich-psychotherapeutischen Methoden durchzuführen ist.

${ }^{2}$ In Österreich werden die anerkannten Ausbildungsrichtungen (psychotherapeutische Schulen) zu vier Verfahrensclustern (Grundorientierungen) zusammengefasst: Psychodynamische Psychotherapie, Humanistische Psychotherapie, Verhaltenstherapie, Systemische Psychotherapie.

3 vgl. §§ 20-22 Psychotherapiegesetz, BGBl 1990/361.
} 
wissenschaftlichen Expert*innenbeirat ${ }^{4}$, der die unterschiedlichen Psychotherapieverfahren repräsentiert. Gemäß ihrer Zielsetzung organisiert die Koordinationsstelle regelmäßig wissenschaftliche Veranstaltungen, Beratungs- und Vernetzungsangebote (Fachtagungen, Workshops) und erstellt entsprechende Publikationen - unter anderem den gegenständlichen Leitfaden (Riess 2018). ${ }^{5}$

\section{Zielsetzung des Leitfadens: Psychotherapie- forschung als verfahrensübergreifend identitätsstiftende wissenschaftliche Grundlage von Psychotherapie}

Der Einfluss und Nutzen von Psychotherapieforschung als Instrument der Weiterentwicklung und Qualitätssicherung von Lehre und klinischer Praxis der Psychotherapie ist hoch. Entsprechend der Vielfalt psychotherapeutischer Ansätze, die in Österreich vertreten sind, unterscheiden sich auch deren $\mathrm{Zu}$ gänge zur Forschung, vor allem in Bezug auf die unterschiedliche Konzeption ihrer Wirksamkeits-/ Erfolgskriterien. Trotz der Vielfalt gibt es jedoch übergreifende Ansätze und Qualitätsmerkmale psychotherapeutischer Forschung. Hier setzt der Leitfaden an: Er hat zum Ziel, Grundlagen und Qualitätsmerkmale von Psychotherapieforschung darzustellen, das wissenschaftliche Selbstverständnis und die gemeinsame Identität von wissenschaftlich fundierter Psychotherapie über Schulen bzw. Verfahren hinweg zu fördern und so Psychotherapieforschung als Kernelement einer forschungsbasierten psychotherapeutischen Wissenschaft sichtbar zu machen.

\section{Entwicklung des Leitfadens}

Der Leitfadenentwicklung gingen seit 2015 mehrere Workshops mit den wissenschaftlichen Beauftragten der psychotherapeutischen Fachspezifika und zahlreiche Diskussionen im Fachbeirat der Koordinationsstelle voraus, in denen die grundsätzliche Zielsetzung des Leitfadens konzipiert wurde. In die Recherchen wurden neben psychotherapiewissenschaftlichen Grundlagenwerken und klassischen sozialwissenschaftlichen Methoden(hand)büchern insbesondere aktuelle wissenschaftliche bzw. wissenschafts-

\footnotetext{
${ }^{4}$ Die Mitglieder des Expert*innenbeirats sind Markus Hochgerner MMsc (Vorsitzender des Ausschusses für fachspezifische Angelegenheiten im Psychotherapiebeirat), Univ.-Prof. Christian Korunka (Universität Wien, Fakultät für Psychologie), Prof. ${ }^{\text {in }}{ }^{D}$. ${ }^{\text {in }}$ Brigitte Schigl (Donau-Universität Krems, Department für Psychotherapie und Biopsychosoziale Gesundheit), Assoc. Prof. ${ }^{\text {in }}$ Henriette Löffler-Stastka (Medizinische Universität Wien, Klinik für Psychoanalyse und Psychotherapie), Assoc. Prof. Dr. Michael Wieser (Universität Klagenfurt, Abteilung für Klinische Psychologie, Psychotherapie und Psychoanalyse) sowie zu Beginn auch Univ.-Ass. Dr. ${ }^{\text {in }}$ Diana Braakmann (SFU), Ao. Univ. Prof. AntonRupert Laireiter (Universität Salzburg), Dr. ${ }^{\text {in }}$ Gerda Metha (SFU). ${ }^{5}$ https://goeg.at/Koordinationsstelle_Psychotherapieforschung.
}

theoretische Diskurse einbezogen. Als Grundlage für die Entwicklung des Stufenplans zur Förderung von Wissenschaft und Forschung in der psychotherapeutischen Ausbildung dienten die Core Competencies of an European Psychotherapist der European Association of Psychotherapy (EAP 2013).

\section{Aufbau, Struktur und Inhalt des Leitfadens}

Teil A - Theorie der Psychotherapieforschung stellt aktuelle wissenschaftstheoretische Reflexionen, Paradigmen, Gütekriterien und Zielsetzungen der (praxisorientierten) Psychotherapieforschung dar. Die relevanten Paradigmen für praxisorientierte Psychotherapieforschung sind das philosophische bzw. formal-geisteswissenschaftliche Paradigma sowie das qualitative und das quantitative Paradigma, die einander ergänzen bzw. in einem spezifischen Verhältnis zueinander $\mathrm{zu}$ sehen sind (Triangulation).

Im Teil B - Praxis der Psychotherapieforschung werden die umsetzungsorientierten Aspekte zu Forschungsansätzen (Theorieorientierte Grundlagenforschung, Wirksamkeitsforschung, Wirkungsforschung, Versorgungsforschung, Ausbildungsforschung), die Forschungsdesigns (experimentelle, quasi-experimentelle und nicht-experimentelle Korrelations-/ Beobachtungsstudien sowie Einzelfallstudien, systematische Fallstudien, Fallvergleichsstudien, phänomenologisches Arbeiten inkl. Grounded Theory etc.) und ausgewählte Forschungsmethoden in ihrer Vielfalt (Methodenpluralismus) sowie Qualitätskriterien für (die Darstellung von) wissenschaftliche(n) Arbeiten anwendungsorientiert aufbereitet. Weiterführende (Literatur-)Hinweise ermöglichen, sich in Planung, Abwicklung und Darstellung von Forschungsprojekten zu orientieren.

Teil C enthält abgeleitete Empfehlungen, die auf einem Stufenplan zur Förderung von Wissenschaft und Forschung in den psychotherapeutischen Ausbildungseinrichtungen basieren. Das Gesundheitsministerium möchte in Zukunft auf allen drei Ebenen die Aktivitäten der psychotherapeutischen Ausbildungsvereine erfassen. Der Stufenplan sieht diese drei Ebenen vor:

- Förderung der rezeptiven Auseinandersetzung mit Psychotherapieforschung

- Förderung des wissenschaftlichen Arbeitens in der Psychotherapieausbildung

- Förderung der aktiven empirischen Psychotherapieforschungstätigkeit

Der Hauptfokus des Leitfadens ist auf praxisorientierte Psychotherapieforschung und insbesondere verfahrensübergreifende, patient*innenorientierte Aspekte gerichtet. Teil B beschreibt, wie die Interessen der Praxis mit den akademischen Anforderungen an Forschung sinnvoll verknüpft werden können. 


\section{Zielgruppen des Leitfadens}

Der Leitfaden dient allen relevanten Organisationsebenen der psychotherapeutischen Ausbildungseinrichtungen als gemeinsame Referenz und Orientierungshilfe, um den Stufenplan Wissenschaft und Forschung im Verfahrenscluster auf allen drei Ebenen umsetzen und verwirklichen zu können. Damit werden insbesondere die Beauftragten für Wissenschaft und Forschung in den Ausbildungseinrichtungen in ihren wissenschaftlichen (Forschungs-)Aktivitäten unterstützt. Der Leitfaden kann als Grundlage ${ }^{6}$ für ein gemeinsames, verfahrensübergreifendes wissenschaftliches Vokabular und für die geplanten Erhebungen, wissenschaftlichen Aktivitäten bzw. die erforderliche Darstellung von Forschungsprojekten verwendet werden.

Für die notwendigen Entwicklungen auf der Organisationsebene (z. B. Adaptierung der Psychotherapieausbildungscurricula) liefert der Leitfaden konkrete Anregungen. In der Psychotherapieausbildung, insbesondere bei der Erstellung bzw. Betreuung wissenschaftlicher Abschlussarbeiten oder Masterthesen, kann er sowohl Auszubildende als auch Lehrende wirkungsvoll unterstützen. Der Leitfaden soll empirische Arbeiten anregen, den Inspirierten die nötigen „handwerklichen“ Grundlagen dafür aufzeigen und sie auf weiterführende Literatur hinweisen.

Praktizierenden Psychotherapeut*innen, die ihre eigenen Erfahrungen und die ihres Arbeitsfeldes durch systematische Erforschung kritisch prüfen wollen, bietet der Leitfaden Einblick in die Forschungslandschaft: Forschungsfragen und Forschungsansätze sowie ein Methodenüberblick zu Erhebung und Auswertung werden auch weniger Wissenschaftserprobten Zugänge eröffnen. Die Brücke zwischen klinisch-praktischer Erfahrung und wissenschaftlicher bzw. Forschungsmethodenkompetenz ist im Sinne des Scientist Practitioner ein erklärtes Ziel der Berufsfeldentwicklung ${ }^{7}$ und zentrale Zielsetzung des Leitfadens.

\section{Psychotherapieforschung als ein eigenständiger Forschungsansatz}

Die zentrale Aussage des Leitfadens ist, dass sich Psychotherapieforschung - mit zahlreichen Berührungspunkten und Querverbindungen zu Nachbarfächern als eigenständige Wissenschaftsdisziplin neben medizinischer und klinisch-psychologischer Forschung etabliert hat. Psychotherapieforschung ist insofern ihrem Gegenstand inhärent, als eine forschende Haltung, die um das Verstehen der Patient*innen bzw. ih-

\footnotetext{
6 im Sinne einer Art Skriptum.

7 zumal die Ausübung der Psychotherapie in Österreich einem unabhängigen, selbstständigen, wissenschaftlich fundierten Heilberuf obliegt mit - europaweit herausragend - reglementierter, aufwendiger Ausbildung.
}

rer Symptome und Leidenszustände bemüht ist, eng verknüpft ist mit der Heilung dieser Leidenszustände - im Sinne eines Junktims von Heilen und Forschen (vgl. Freud 1909; Jandl-Jager 1997). Psychotherapieforschung besteht also seit der Entstehung der Psychotherapie und entwickelt sich stetig weiter. Psychotherapieforschung ist charakterisiert durch eine umfassende wissenschaftstheoretische Verankerung, die weit über den verkürzten Begriff der Evidenzbasierung hinausgeht.

\section{Kritische Gegenstandsbestimmung, Multiparadigmatik und Methodenpluralismus in der Psychotherapieforschung}

Der Leitfaden arbeitet folgende zentrale Prämissen heraus:

Die Basis jeder sinnvollen Psychotherapieforschung ist eine kritische Gegenstandsbestimmung, v.a. in Bezug auf einen unverkürzten Subjektbegriff in seinem spezifischen Kontext. Psychotherapieforschung gründet im Verständnis des Menschen als bio-psychosoziales Subjekt: Ihr Gegenstand ist das intentionale Subjekt in seinem konkreten, historisch-gesellschaftlichen Kontext. Dieses Subjekt bezieht sich fühlend, wollend, denkend auf seine soziale Umgebung bzw. die Welt und befindet sich in einem kontinuierlichen dynamischen Entwicklungsprozess, der einer spezifischen Veränderungslogik folgt (vgl. Fischer und Eichenberg 2011). Der Kontext ist mitverantwortlich für die hohe Komplexität des Untersuchungsgegenstandes in der Psychotherapieforschung (vgl. Orlinsky und Howard 1987; Wampold und Imel 2015) und muss im Forschungsdesign mitberücksichtigt werden.

Um also ihrem Gegenstand angemessene, wissenschaftlich gültige Aussagen zu produzieren, muss Psychotherapieforschung zweitens die Prinzipien der logisch-empirischen Konvergenz (Gegenstandsadäquatheit), die eine logisch-apriorische Begriffsanalyse voraussetzt, und der methodisch-empirischen Konvergenz umsetzen (vgl. Fischer und Eichenberg 2011). Diese spezifische wissenschaftstheoretische Position impliziert einen multiparadigmatischen und methodenpluralistischen Forschungsansatz: Der komplexe Gegenstand der Psychotherapieforschung mit seiner spezifischen Veränderungslogik ist nur in einer $\mathrm{Zu}$ sammenschau unterschiedlicher wissenschaftlicher Methodologien fassbar und erforschbar (vgl. Bergin et al. 2013; Döring und Bortz 2016; Gelo et al. 2015).

Drittens entwickelt sich der idealtypische Gegensatz zwischen quantitativen und qualitativen Forschungsansätzen bzw. philosophisch-theoretischen Ansätzen zunehmend zu einem Ergänzungsverhältnis im Sinne der Triangulation (Gahleitner et al. 2014). Dabei gilt aber in der Psychotherapieforschung letztlich das Primat des Qualitativen (bzw. des philosophisch-theoretischen Ansatzes), denn jeder empirischen Untersuchung geht der Aspekt des Qualitativen logisch voraus: Vor jeder quantifizie- 
renden empirischen Untersuchung muss qualitativ bzw. theoretisch-logisch präzise definiert werden, was ggf. zu messen ist, ganz im Sinne der logischapriorischen Begriffsanalyse (vgl. Fischer und Eichenberg 2011). Insofern ist Quantifizierung eine Hilfswissenschaft für den qualitativ-theoretischen Aspekt in der Psychotherapieforschung (vgl. Döring und Bortz 2016). Dabei sind Subjektivität und Kontext keine „Störfaktoren“, sondern müssen forschungslogisch mitberücksichtigt werden. Eine Reduktion des Subjekts als Forschungsgegenstand etwa auf die Summe von Merkmalsausprägungen, isolierte Variablen, biologisch-chemisch-physikalische Gesetzmäßigkeiten des Gehirns oder statistische Normabweichung wäre - unausgewiesen und hinsichtlich des Nutzens unbegründet - nicht zulässig (Reduktionismusproblem) (vgl. Kimmerle 2012; Schülein 2012).

\section{Ausblick}

Der Leitfaden will interessierte Praktiker*innen für Psychotherapieforschung begeistern - ganz im Sinne des Scientist Practitioner. Mögen ihre Ergebnisse vor allem hinsichtlich des Nachweises positiver Effekte auf die psychische Gesundheit durch Psychotherapie - auch verantwortliche Entscheidungsträger*innen im Versorgungssystem überzeugen.

Der Leitfaden ist unter abrufbar: https://jasmin. goeg.at/420/1/Leitfaden_Praxisorientierte_Psychothe rapieforschung.pdf.

Interessenkonflikt G. Riess gibt an, dass kein Interessenkonflikt besteht.

\section{Literatur}

Bergin, A. E., Garfield, S. L., \& Lambert, M. J. (2013). Handbook of psychotherapy and behavior change (6. Aufl.). New Jersey: John Wiley \& Sons.
Döring, N. \& Bortz, J. (2016). Forschungsmethoden und Evaluation in den Sozial- und Humanwissenschaften (5.Aufl.). Berlin: Springer.

EAP (2013). The core competencies of A European psychotherapist. The professional competencies of a European psychotherapist-A project of the European association of psychotherapy (EAP).: European Association of Psychotherapy.

Fischer, G., \& Eichenberg, C. (2011). Psychotherapiewissenschaft: Einführung in eine neue humanwissenschaftliche Disziplin. Gießen:Psychosozial-Verlag.

Freud, S. (1909). Analyse der Phobie eines Fünfjährigen Knaben - Der Kleine Hans. Freud-Studienausgabe, Bd. 8. Frankfurt: Fischer.

Gahleitner, S., Schmitt, R., \& Gerlich, K. (2014). Qualitative und Quantitative Forschungsmethoden - für EinsteigerInnen aus den Arbeitsfeldern Beratung, Psychotherapie und Supervision. Weitramsdorf-Weidach:ZKS.

Gelo, O.C. G., Pritz, A., \& Rieken, B. (2015). Psychotherapy research. Foundations, process and outcome. Wien: Springer.

Jandl-Jager, E., et al. (1997). Heilen und Forschen. Psychotherapie Forum, 5(1), 3.

Kimmerle, G. (2012). Am Beispiel Freud. Wahrheitsvoraussetzungen in der Wissenschaftsgeschichte. Psyche Zeitschrift für Psychoanalyse und ihre Anwendungen, 66(7), 638-648.

Orlinsky, D.E., \& Howard, K.I. (1987). A generic model of psychotherapy. Journal of Integrative \& Eclectic Psychotherapy, 6, 6-27.

Riess, G. (2018). Praxisorientierte Psychotherapieforschung. Leitfaden zur Förderungvon WissenschaftundForschung in der psychotherapeutischen Ausbildung. Gesundheit Österreich GmbH, Wien. https://jasmin.goeg.at/420/1/ Leitfaden_Praxisorientierte_Psychotherapieforschung. pdf.Zugegriffen: 4. Mai 2021.

Schülein, J.A. (2012). „Ewige Jugend“ - Warum psychoanalytische Theorie die Probleme hat, die sie hat. Psyche Zeitschrift für Psychoanalyse und ihre Anwendungen, 66(7), 606-637.

Wampold, B.E., \& Imel, Z.E. (2015). The great psychotherapy debate: The evidence for what makes psychotherapy work. New York: Routledge.

Hinweis des Verlags Der Verlag bleibt in Hinblick auf geografische Zuordnungen und Gebietsbezeichnungen in veröffentlichten Karten und Institutsadressen neutral. 